\title{
Prieurianin Causes Weight Loss in Diet-Induced Obese Mice and Inhibits Adipogenesis in Cultured Preadipocytes
} \author{
Khew-Voon Chin ${ }^{1,4,5^{*}}$ \\ ${ }^{1}$ Department of Medicine, University of Toledo, College of Medicine, Toledo, $\mathrm{OH}$, United States \\ ${ }^{2}$ Department of Surgery, University of Toledo, College of Medicine, Toledo, $\mathrm{OH}$, United States \\ ${ }^{3}$ Department of Obstetrics and Gynecology, National Defense Medical College, Tokorozawa, Saitama, Japan \\ ${ }^{4}$ Center for Diabetes and Endocrine Research, University of Toledo, College of Medicine, Toledo, OH, United States \\ ${ }^{5}$ Department of Biochemistry and Cancer Biology, University of Toledo, College of Medicine, Toledo, OH, United States \\ ¥These authors contributed equally to this work
}

Ahmed Kablan ${ }^{1 \ddagger}$, Rudel A.Saunders ${ }^{1 \ddagger}$, Maria Szkudlarek-Mikho ${ }^{1}$, Andrew J.B. Chin ${ }^{1}$, Raul M. Bosio ${ }^{2}$, Kazuyuki Fujii ${ }^{1,3}$, Joseph Shapiro ${ }^{1}$ and

\begin{abstract}
The global increase in the incidence of obesity has emerged as one of the most serious public health risks in recent years. Despite the enormity of the obesity pandemic, there are currently only two FDA-approved therapies for its treatment and these drugs exhibit modest efficacy and have limiting side effects. Prieurianin is a plant limonoid product that deters feeding in insect larvae. We investigated in this study the effects of prieurianin on weight loss and adipogenesis. Our results showed that prieurianin causes weight loss by reducing energy intake in obese mice on highcalorie diet. We also found that prieurianin is anti-adipogenic in cultured preadipocytes and adipocytes by inhibiting proliferation and differentiation of preadipocytes into adipocytes, and induces either dedifferentiation or delipidation of mature adipocytes. Whether prieurianin can potentially be used for obesity treatment in human warrants further investigation.
\end{abstract}

Keywords: Prieurianin; Obesity; Appetite; Adipogenesis; Weight loss; Tolerance; Preadipocytes; Adipocytes

\section{Introduction}

Thealarming riseintheincidenceofobesityworldwidehasemerged as one of the most pressing public health issu es in recent years [1]. The obesity pandemic is thought to be associated with a sedentary lifestyle and the overconsumption of energy-rich food. It is clear that obesity raises the incidence of some co-morbid health risks such as diabetes, hypertension, cardiovascular and metabolic diseases [2]. Despite the enormity of the obesity pandemic and the wealth of information that has been gained in the past decade about the hormonal and transcriptional mechanisms that regulate energy metabolism, there are only two drugs currently approved by the FDA for the treatment of obesity.These include orlistat that blocks the absorption of dietary fat [3], and sibutramine, a specific reuptake inhibitor for norepinephrine and serotonin that acts in the central nervous system (CNS) to reduce energy intake [4]. These drugs have limited efficacies and side effects are commonly reported [5], which are further confounded by diminishing response in the long-term treatment of obesity [6,7]. The loss of efficacy, which may be a result of drug induced tolerance, not only occurs with the first generation appetite suppressant but are observed with the other classes of drug candidates as well including rimonabant [8], leptin [9], and melanotan II [10]. Moreover, anti-obesity drug development strategy continues to focus on either central or peripheral acting inhibitors of food intake [11], which will likely encounter similar problems [6]. Recent suspension of sibutramine in Europe, and the FDA-issued warning of heart risk in patients taking sibutramine further limit the pharmacotherapeutic option for obesity.

The completion of some recent late stage anti-obesity clinical trials using combinations of various repositioned drugs including anticonvulsant, antidepressant and a controlled substance of the amphetamine class, seemed to meet FDA criteria for clinical efficacy [12]. Other than the novel monotherapeutic lorcaserin, these repositioned drugs, however, all have a history of safety issues. The potential side effects associated with the long-term use of these agents remain to be evaluated. Despite these progresses, it is imperative to identify new drugs to address the unmet needs for the treatment of obesity.

Some compounds in plants have been developed for their use in insect pest management without harming the environment [13]. Prieurianin, a limonoid isolated from several plant species, exhibits specific anti-feedant activity in insect using a diet choice bioassay [14]. The drug has no apparent effects on the efficiency of conversion of ingested food in larvae and seems to antagonize 20 -hydroxyecdysone activity in Drosophila cells $[14,15]$.

In light of its ability to deter feeding in insects, we hypothesized that prieurianin would interfere with feeding behaviour in mice and cause weight loss. In this report, we showed that prieurianin suppresses appetite and causes weight loss in diet-induced obese mice and inhibits adipogenesis in cell culture model of preadipocytes. Our results suggest that prieurianin may be an effective anti-obesity drug candidate and also serve as a pharmacological tool for probing the biology of adipose tissue.

\section{Materials and Methods}

Animals, diets, drugs, and reagents

All animal procedures were approved by the Institutional Animal Care and Use Committee. For all in vivo efficacy studies, prieurianin was dissolved in DMSO and then given intraperitoneally as a mixture in $10 \%$ Cremophor EL (Sigma-Aldrich, St. Louis, MO) [16]

*Corresponding author: Khew-Voon Chin, Ph.D, Department of Medicine, University of Toledo, College of Medicine, Toledo, OH, United States, Tel: 419-3833502; Fax: 419-383-4473; E-mail: khew-voon.chin@utoledo.edu

Accepted September 28, 2010; Published September 29, 2010

Citation: Kablan A, Saunders RA, Szkudlarek-Mikho M, Chin JB, Bosio RM, et al (2010) Prieurianin Causes Weight Loss in Diet-Induced Obese Mice and Inhibits Adipogenesis in Cultured Preadipocytes. J Diabetes Metab 1:101. doi:10.4172/21556156.1000101

Copyright: ( $) 2010$ Kablan A, et al. This is an open-access article distributed under the terms of the Creative Commons Attribution License, which permits unrestricted use, distribution, and reproduction in any medium, provided the original author and source are credited. 
to the animals, and vehicle-treated controls received equivolume injections of $0.5 \%$ of DMSO in Cremophor. For diet-induced obesity studies, 12-14 week-old normal C57BL/6 (B6) mice (The Jackson Laboratory, Bar Harbor, ME) were acclimatized for at least one week on a 12 -hour light/dark cycle at $68-72^{\circ} \mathrm{F}$ and then fed a high-fat diet containing $45 \mathrm{kcal} \%$ fat (Research Diets, Inc., New Brunswick, $\mathrm{NJ}$ ), until their mean body weight reached approximately $30 \mathrm{~g}$, and remained on the high-fat diet for the duration of the study. Mice had access to the high fat diet ad libitum during treatment in all studies. Blood glucose and insulin levels were measured and adipose tissues were harvested for weighing at the end of the experiments. Food intake and body weight were measured every two days for the duration of the study. Mice were euthanized by $\mathrm{CO}_{2}$ asphyxiation according to the Association for Assessment and Accreditation of Laboratory Animal Care International guidelines. All studies were performed with at least 10 to 20 animals per group.

Prieurianin was obtained from MicroSource Discovery Systems (Gaylordsville, CT), and sibutramine from Sigma-Aldrich (St. Louis, MO). Tumor necrosis factor $\alpha$ was cloned into the pGEX-6P-1 vector (GE Healthcare, Piscataway, NJ) as a fusion protein with glutathione Stransferase and produced from E. coli following IPTG induction and purification as described according to manufacturer's specification.

\section{Cell culture and adipocyte differentiation}

NIH-3T3/L1 (L1) (American Type Culture Collection, Manassas, VA) and OP9 stromal (a gift of Dr. Perry Bickel, University of Texas Health Science Center, Houston, TX) cells were cultured at $37^{\circ} \mathrm{C}$ with $10 \% \mathrm{CO}_{2}$ in Dulbecco's modified Eagle's medium (DMEM) (Invitrogen, Carlsbad, CA) supplemented with $10 \%(\mathrm{v} / \mathrm{v})$ fetal calf serum (Invitrogen), 1 $\mathrm{mM}$ sodium pyruvate, $0.1 \mathrm{mM}$ non-essential amino acids, 2 $\mathrm{mM}$ L-glutamine, $100 \mu \mathrm{g} \mathrm{m} \mathrm{m}^{-1}$ streptomycin sulfate, and 100 $\mathrm{U} \mathrm{ml} \mathrm{m}^{-1}$ penicillin. To assess preadipocytes proliferation, cells were plated in 12 well dishes and then treated with various concentrations of prieurianin. Cell growth was measured daily on a Coulter cell counter. To differentiate L1 cells into adipocytes, cells were incubated with $250 \mathrm{nM}$ dexamethasone, $450 \mu \mathrm{M}$ 3-isobutyl-1-methylxanthine, and $167 \mathrm{nM}$ insulin for 2 days, followed by $167 \mathrm{nM}$ insulin for an additional 3 days as described (17). For OP9 cells (18), differentiation was initiated with a serum replacement medium composed of MEM-a with 15\% KnockOut SR (Invitrogen,), $100 \mu \mathrm{g} \mathrm{ml}{ }^{1}$ streptomycin sulfate, and $100 \mathrm{U} \mathrm{ml}^{-1}$ penicillin, for 2 days and then replenished in the propagation medium as above. Nile Red (Sigma, St. Louis, MO) staining following differentiation was performed as described by adding a $1 \mathrm{mg} \mathrm{ml}^{-1}$ stock solution to cultured cells to a final concentration of $5 \mu \mathrm{g} \mathrm{ml}^{-1}$ and then visualized under fluorescence microscope [19], and compared to preadipocytes differentiated in the presence of prieurianin. To determine the effect of prieurianin on dedifferentiation/lipolysis, preadipocytes were differentiated into adipocytes as above and further cultured for 5-6 days, and then treated with or without drug for an additional 5-6 days before Nile Red staining.

\section{Annexin V assay}

The Guava Nexin assay measures membrane changes associated with apoptosis using Annexin V-PE, and 7-aminoactinomycin D (7-AAD) to identify dead cells, and experiments were conducted according to manufacturer's specification (Millipore, Billerica, MA). In brief, preadipocytes in culture were treated with prieurianin for 24 hours and then resuspended in 1X Nexin buffer solution, supplemented with $5 \mu \mathrm{l}$ of Annexin-V solution and $5 \mu \mathrm{l}$ of 7-AAD. To ensure the binding of the label, samples were incubated on ice. Finally, the 1X Nexin buffer was added and samples were analyzed in the Guava cytometer.

\section{Glucose, insulin and adipose tissues}

Fed glucose and insulin levels were measured from blood samples taken at the end of the treatment period. Glucose was measured using Cardio Chek meter (PTS, Indianapolis, IN). Insulin levels were measured in plasma using an ELISA kit (Crystal Chem Inc., Downers Grove, IL). Necropsy analysis was performed for two fat depots, subcutaneous and visceral fat, by weighing the dissected tissues.

\section{Statistical analyses}

Statistical analyses were performed using commercially available software package (PRISM, GraphPad Software, Inc., La Jolla, CA) or the data analysis add-in for Microsoft Excel. Either single factor ANOVA or Student's t-tests were used for all analysis as indicated. P values of less than 0.05 were considered statistically significant.

\section{Results}

Anorexigenicity. The observation that prieurianin (Figure1A) is a feeding deterrent in insect larvae that presumably acts by

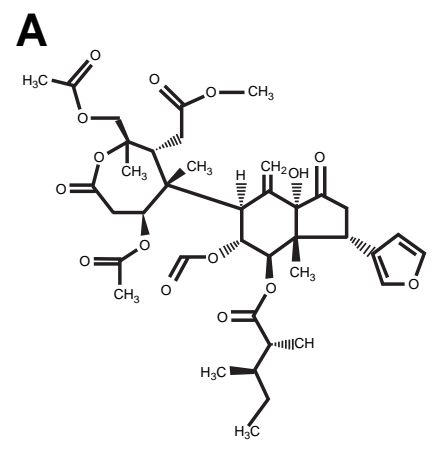

B

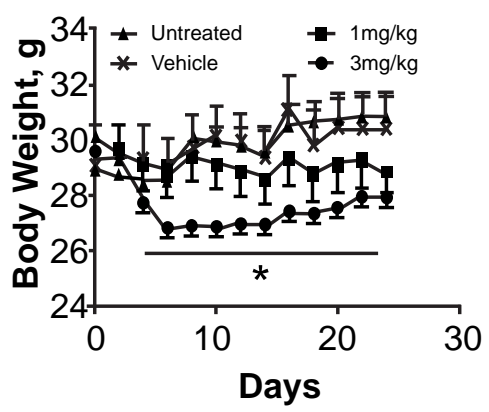

C

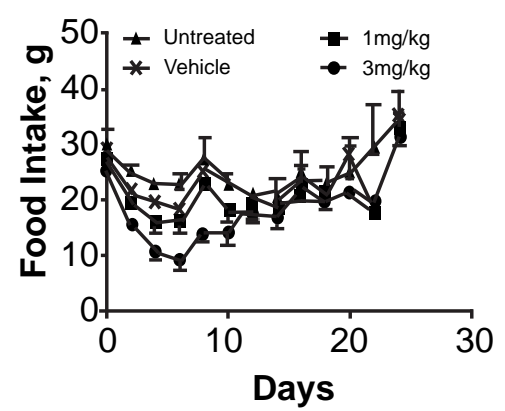

Figure 1: Prieurianin in mouse model of diet-induced obesity (DIO). A, Structure of prieurianin. B, C, Body weight and amount of food consumed by DIO B6 mice that were given either 1 or $3 \mathrm{mg} \mathrm{kg}^{-1}$ of prieurianin for three weeks. Prieurianin causes weight loss (B) and reduced food consumption (C) in DIO B6 mice compared to untreated and vehicle-treated controls. All studies consisted of ten mice per group. Statistics were conducted as an ANOVA; asterisk, $\mathrm{P}<0.05$ versus untreated and vehicle-treated controls. Error bars indicate s.e.m. 


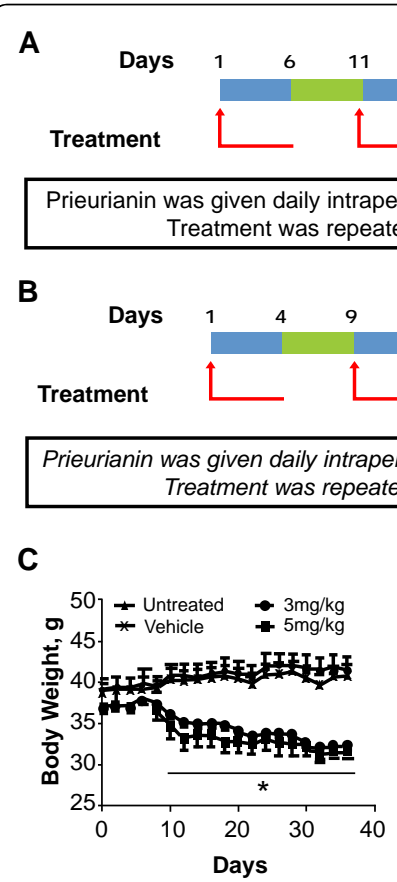

Figure 2: Effects of drug holiday schedule for prieurianin in DIO mice. A, B, Drug holiday treatment protocols for prieurianin in DIO mice. This on-off or cyclical treatment strategy comprised of specified doses of prieurianin at either 3 or $5 \mathrm{mg} \mathrm{kg}^{-1}$ were given as indicated for a defined duration coupled with intermittent drug holiday to overcome drug-induced tolerance. C, D, Drug holiday treatment schedule with either 3 or $5 \mathrm{mg} \mathrm{kg}^{-1}$ prieurianin circumvented drug-induced tolerance and produced a sustained weight loss (C) and reduced food intake (D). All studies consisted of 10 animals per group, except for the 3 and $5 \mathrm{mg} \mathrm{kg}^{-1}$ prieurianin treated groups $(n=20)$.

antagonizing 20-hydroxyecdysone in Drosophila cells prompted us to ask whether the anti-feedant effects of prieurianin can be exploited for the treatment of obesity. To test this hypothesis, we examined the effects of prieurianin on weight loss in the dietinduced obese (DIO) C57BL/6 (B6) mice, since this model has been widely used to investigate the underlying mechanisms of obesity in human [20,21]. DIO B6 mice were given high fat diet for 15 weeks and then prieurianin was administered intraperitoneally (i.p.) daily with either 1 or $3 \mathrm{mg} \mathrm{kg}^{-1}$ prieurianin for three weeks. Prieurianin caused a dose-dependent weight loss (Figure1B) that was accompanied by $70-80 \%$ decrease in food consumption, which eventually returned to normal levels by the end of the treatment period (Figure 1C). Notably, a gradual regain in body weight was observed following the first week of treatment (Figure1B), suggesting the onset of druginduced tolerance.

One of the major unresolved problems in the pharmacotherapy of obesity is the diminishing efficacy of drugs with chronic treatment [6]. To assess whether drug-induced tolerance can be circumvented by "drug holidays", mice were treated with either 3 or $5 \mathrm{mg} \mathrm{kg}^{-1}$ prieurianin for 5 or 3 days, respectively, followed by 5 days of drug withdrawal, and repeating this regimen for 4 cycles (Figure $2 \mathrm{~A}$ and B). DIO B6 mice from the experiments in (Figure1) were maintained on the high calorie diet for four additional weeks without treatment and then subjected to the drug holiday treatment cycle. This treatment protocol, at either 3 or $5 \mathrm{mg} \mathrm{kg}^{-1}$ prieurianin, produced a greater response than daily treatment, with up to $20 \%$ weight loss at either dosage, and no observable weight regain at the end of the treatment cycle (Figure 2C). Food consumption decreased

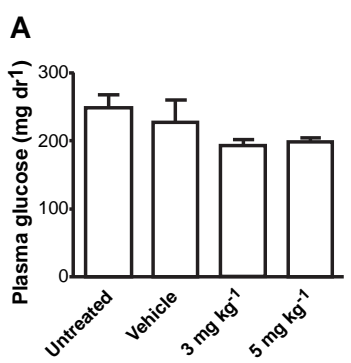

C

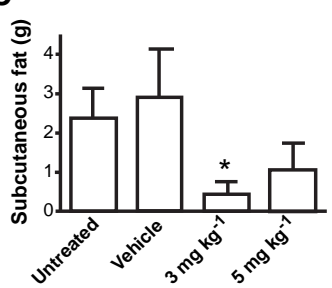

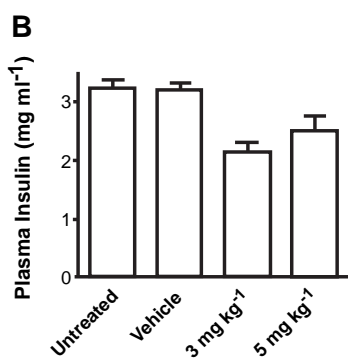

D

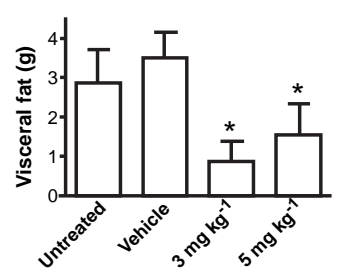

Figure 3: Effects of prieurianin on plasma glucose and insulin, and adipose mass in DIO mice. Plasma glucose (A) and insulin (B) levels were measured at the end of prieurianin treatment. Prieurianin treatment decreased plasma glucose (filled bar) and insulin (opened bar) levels in DIO mice. C, D, Significant reduction in adipose mass was observed in post-mortem analysis of in subcutaneous (C) and visceral (D) compartments of prieurianin treated DIO B6 mice compared to untreated and vehicle (DMSO)-treated controls. All studies consisted of 10 animals per group, except for the 3 and $5 \mathrm{mg} \mathrm{kg}^{-1}$ treated groups $(n=20)$ in $\mathbf{A}$ and $\mathbf{B}$. Experiments were conducted in triplicates for $\mathbf{C}$ and $\mathbf{D}$ selected randomly from the animals. Statistics were conducted as an ANOVA; asterisk, $\mathrm{P}<0.05$ versus untreated and vehicle-treated controls. Error bars indicate s.e.m.

precipitously initially, but gradually returned to approximately 60 $70 \%$ of normal feeding and maintained at that level for the duration of the treatment cycle (Figure 2D). Fed glucose and insulin levels also showed a trend of decrease at the end of treatment period (Figure $3 \mathrm{~A}$ and $\mathrm{B}$ ).

\section{Anti-adipogenesis}

Post-mortem analysis showed $>50 \%$ decrease in subcutaneous and visceral fat depots in prieurianin-treated DIO B6 mice (Figure $3 \mathrm{C}$ and $\mathrm{D}$ ), compared to untreated and vehicle-treated controls. The ability of prieurianin to reduce fat mass led us to determine the effects of prieurianin on the differentiation of cultured preadipocytes into mature adipocytes using either the L1 preadipocytes [17], or the OP9 mouse stromal cells [18] that are capable of differentiating into adipocytes. Prieurianin inhibited the proliferation of $\mathrm{L} 1$ preadipocytes in a dose-dependent manner (Figure 4A), as well as their post-confluent mitosis and clonal expansion, evident from the reduced ${ }^{3}[\mathrm{H}]$ thymidine uptake following differentiation induction (Figure 4B). Prieurianin also inhibited the differentiation of OP9 stromal cells into adipocytes as evident from the reduced Oil Red $O$ stained lipid-accumulating adipocytes relative to the untreated/undifferentiated and the differentiated controls (Figure $5 \mathrm{~A})$. The positive control, TNF $\alpha[22,23]$, also inhibited OP9 cells differentiation (Figure 5A). These effects of prieurianin were recapitulated in the $L 1$ preadipocytes (data not shown). The decrease in adipocytes was not a result of prieurianin-induced apoptosis, as indicated by the lack of annexin $\mathrm{V}$ binding to phosphatidylserine, in contrast to stromal cells treated with doxorubicin, a cytotoxic drug that causes apoptosis (Figure 4C). Similarly, apoptosis was not observed in prieurianin-treated L1 preadipocytes (data not shown). 

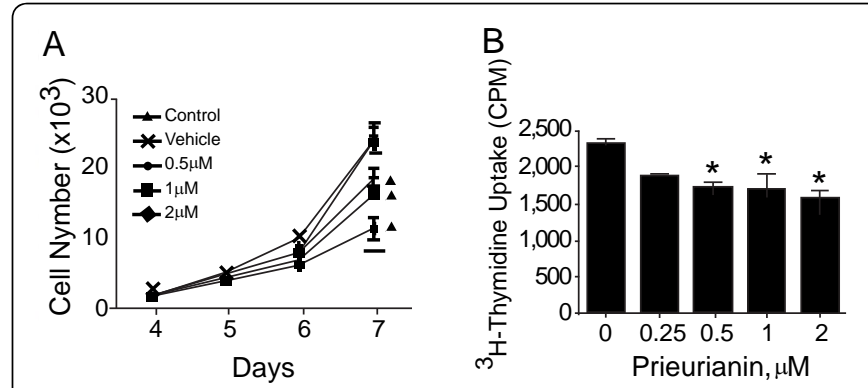

C

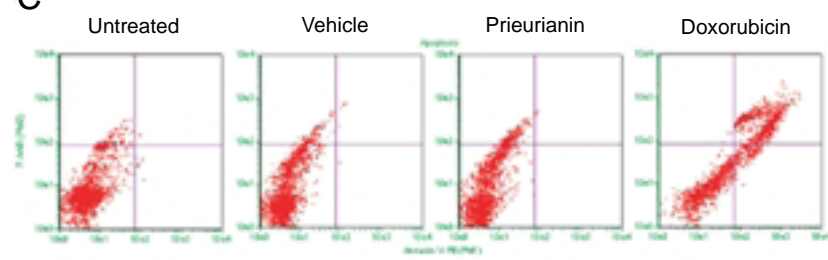

Figure 4: Effects of prieurianin on L1 preadipocytes and OP9 stromal cells. ( A), L1 preadipocytes were cultured in 12-well dishes and counted daily using a Coulter cell counter. Prieurianin inhibits L1 preadipocytes proliferation. (B), L1 preadipocytes were cultured to confluence and then placed in a differentiation cocktail for 48 hours followed by ${ }^{3} \mathrm{H}$-thymidine uptake and cell lysates were subjected to scintillation counting. Prieurianin inhibits the postconfluent mitosis and clonal expansion of L1 preadipocytes. (C), Prieurianin does not cause apoptosis in OP9 cells as measured by annexin $\checkmark$ binding to cell surface phosphatidylserine, compared to the cytotoxic drug, doxorubicin. Studies were performed in triplicates. Asterisk, $\mathrm{P}<0.05$ versus untreated controls. Error bars indicate s.e.m.

To assess the effects of prieurianin in mature adipocytes, L1 preadipocytes were differentiated into adipocytes followed by treatment with either TNF $\alpha$ or prieurianin. Consistent with previous report [22], TNF $\alpha$ induced rapid lipolysis, resulting in significant loss of Nile Red stained adipocytes (Figure 5B). Though less potent, prieurianin also caused a loss of lipid in the adipocytes, marked by reduced Nile Red positive cells compared to differentiated control and vehicle-treated cells (Figure 5B).

\section{Discussion}

Despite a growing obese population worldwide, pharmacotherapy for obesity is limited as there are currently only two FDA-approved drugs for its treatment in U.S. Recent FDA issued heart risk warning in U.S. for sibutramine and its withdrawal in Europe, as well as new concerns for potential liver toxicity with orlistat, further reduced the armamentarium for the combat of obesity. Moreover, the use of repositioned drugs comprising of combinatorial mixture of anticonvulsant, antidepressant, or psychoactive stimulant, also poses questions of unknown longterm safety concerns, as these drugs all have various known risks and side effects [24]. These issues all point to an urgent need for the development of more efficacious and safe pharmacotherapeutics for obesity.

Our studies here showed that prieurianin, a plant-derived limonoid product, which exhibits feeding deterrent activity in insect larvae, has biological activity in high-fat diet induced obese mice that results in appetite suppression, leading to decreased energy intake in mice and weight loss. Further, prieurianin also inhibits the proliferation and differentiation of preadipocytes, as well as causing mature adipocytes to lose their lipid content. These findings suggest a novel anti-obesity drug candidate characterized by a dual anorexigenic and anti-

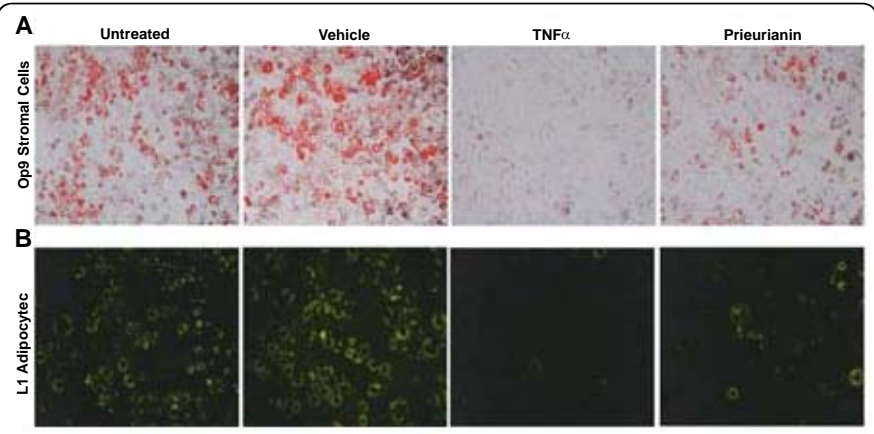

Figure 5: Prieurianin inhibits adipogenesis. (A), Prieurianin inhibits the differentiation of OP9 cells into adipocytes, stained with Oil Red O. TNFa, a cytokine and potent inhibitor of differentiation, is used as control. (B), Prieurianin causes either dedifferentiation or delipidation of differentiated adipocytes, stained with Nile Red, compared to untreated and vehicletreated adipocytes. TNF $\alpha$, which causes lipolysis, serves as control. All experiments were performed for at least three or more times and representative micrographs of the experiments were shown.

adipogenic property that is distinct from currently available pharmaceutical therapies and pipeline products in development that focused on either suppressing satiety or inhibiting energy homeostasis [11]. The anorexigenic effect of prieurianin could not have been due to taste aversion because the drug was delivered by IP injection into the mice. Moreover, the diet choice bioassay in insect larvae also suggests a specific effect of prieurianin on feeding stimulus [14]. Although it was shown to antagonize 20-hydroxyecdysone action in Drosophila cells [15], the precise mechanism of action of prieurianin, however, is unknown with respect to its effects on appetite suppression in mice and its inhibition of adipogenesis in cultured preadipocytes. It is noteworthy that prieurianin was recently shown to inhibit endocytosis and also block brassinosteroid-induced gene expression in germinating seedlings of Arabidopsis thaliana [25]. It is unclear how prieurianin-inhibited endocytosis translates into its antiobesity effects. Furthermore, the inhibition of hormone-induced transcription in germinating seedlings by prieurianin suggests that the drug may have similar effects on the transcriptional regulation of adipogenesis in preadipocytes by disrupting gene expression program during differentiation. Hence, identifying the molecular target(s) of prieurianin might yield insights into its anorexigenic activity in suppressing energy intake and its anti-adipogenic potential in the development of adipose tissue.

Long-term efficacy and effectiveness of obesity treatments are known to have poor outcome [26]. The potential of druginduced tolerance associated with anti-obesity therapeutics is long recognized, but circumventing its development has not been fully addressed [6]. Our studies here demonstrated that drug-induced tolerance resulting from chronic pharmacotherapy for obesity could be overcome by a cyclical drug holiday treatment strategy, which produces a greater and more durable therapeutic response as evident by the sustained weight loss and reduced energy intake. Hence, the on-off treatment protocol may potentially restore the efficacy of currently available anti-obesity drugs as well as other experimental pharmacotherapeutics that are prone to drug-induced tolerance in the long-term treatment of obesity. Besides, decreasing the frequency of drug exposure using the drug holiday treatment protocol may reduce the incidence and disposition to the toxicities of these anti-obesity drugs. 
Citation: Kablan A, Saunders RA, Szkudlarek-Mikho M, Chin JB, Bosio RM, et al. (2010) Prieurianin Causes Weight Loss in Diet-Induced Obese Mice and Inhibits Adipogenesis in Cultured Preadipocytes. J Diabetes Metab 1:101. doi:10.4172/2155-6156.1000101

Page 5 of 5

Taken together, the findings in this study showed that prieurianin is a novel anti-obesity therapeutic candidate that exhibits a dual anorexigenic and anti-adipogenic property. Therefore, investigating the precise molecular target of prieurianin, and characterizing its long-term efficacy and toxicology profile will determine its suitability for further development as a pharmacotherapeutic for obesity.

\section{Acknowledgements}

We thank P. Bickel (University of Texas Health Science Centre, Houston) for providing the OP9 stromal preadipocytes. This research is supported in part by National Institutes of Health grant R01 CA102204.

\section{References}

1. Swinburn B, Sacks G, Ravussin E (2009) Increased food energy supply is more than sufficient to explain the US epidemic of obesity. Am J Clin Nutr 90: 1453-1456.

2. Bray GA, Bellanger T (2006) Epidemiology, trends, and morbidities of obesity and the metabolic syndrome. Endocrine 29: 109-117.

3. Guerciolini R (1997) Mode of action of orlistat. Int J Obes Relat Metab Disord 3: S12-23.

4. Ryan DH, Kaiser P, Bray GA (1995) Sibutramine: a novel new agent for obesity treatment. Obes Res 4: 553S-559S

5. Bray GA (2008) Lifestyle and pharmacological approaches to weight loss: efficacy and safety. J Clin Endocrinol Metab 93: S81-88.

6. Fernstrom JD, Choi S (2007) The development of tolerance to drugs that suppress food intake. Pharmacol Ther 117: 105-122.

7. Li Z, Maglione M, Tu W, Mojica W, Arteburn D, Shugarman LR, et al. (2005) Meta-analysis: pharmacologic treatment of obesity. Ann Intern Med142: 532546.

8. Pi-Sunyer FX, Aronne LJ, Heshmati HM, Devin J, Rosenstock J (2006) Effect of rimonabant, a cannabinoid-1 receptor blocker, on weight and cardiometabolic risk factors in overweight or obese patients: RIO-North America: a randomized controlled trial. JAMA 295: 761-775.

9. Sahu A (2002) Resistance to the satiety action of leptin following chronic central leptin infusion is associated with the development of leptin resistance in neuropeptide $Y$ neurones. J Neuroendocrinol 14: 796-804.

10. Seeley RJ, Burklow ML, Wilmer KA, Matthews CC, Reizes O, et al. (2005) The effect of the melanocortin agonist, MT-II, on the defended level of body adiposity. Endocrinology 146: 3732-3738.

11. Cooke D, Bloom S (2006) The obesity pipeline: current strategies in the development of anti-obesity drugs. Nat Rev Drug Discov 5: 919-931.
12. Jones $D$ (2009) Novel pharmacotherapies for obesity poised to enter market. Nat Rev Drug Discov 8: 833- 834 .

13. Isman MB (2006) Botanical insecticides, deterrents, and repellents in modern agriculture and an increasingly regulated world. Annu Rev Entomol 51: 45-66

14. Koul O, Daniewski WM, Multani JS, Gumulka M, Singh G (2003) Antifeedant effects of the limonoids from Entandrophragma candolei (Meliaceae) on the gram pod borer, Helicoverpa armigera (Lepidoptera: Noctuidae). J Agric Food Chem 51: 7271-7275.

15. Sarker SD, Savchenko T, Whiting P, Sik V, Dinan L (1997) Two limonoids from Turraea obtusifolia (Meliaceae), prieurianin and rohitukin, antagonize 20- hydroxyecdysone action in a Drosophila cell line. Arch Insect Biochem Physiol 35: 211-217.

16. Dorr RT (1994) Pharmacology and toxicology of Cremophor EL diluent. Ann Pharmacother 28: S11-14

17. Green H, Meuth M (1974) An established pre-adipose cell line and its differentiation in culture. Cell 3: 127-133.

18. Wolins NE, Quaynor BR, Skinner JR, Tzekov A, Park C et al. (2006) OPg mouse stromal cells rapidly differentiate into adipocytes: characterization of a useful new model of adipogenesis. J LipidRes 47: 450-460.

19. Gonzales AM, Orlando RA (2007) Role of adipocyte-derived lipoprotein lipase in adipocyte hypertrophy. Nutr Metab 4: 22

20. Tschop M, Heiman ML (2001) Rodent obesity models: an overview. Exp Clin Endocrinol Diabetes 109: 307-319.

21. Collins S, Martin TL, Surwit RS, Robidoux J (2004) Genetic vulnerability to diet-induced obesity in the C57BL/6J mouse: physiological and molecular characteristics. Physiol Behav 8: 243-248.

22. Simons PJ, van den Pangaart PS, van Roomen CP, Aerts JM, Boon L (2005) Cytokine-mediated modulation of leptin and adiponectin secretion during in vitro adipogenesis: evidence that tumor necrosis factor-alpha-and interleukin-1beta-treated human preadipocytes are potent leptin producers. Cytokine 32: 94-103.

23. Spiegelman BM, Hotamisligil GS (1993) Through thick and thin: wasting obesity and TNF alpha. Cell 73: 625-627.

24. Robinson JR, Niswender KD (2009) What are the risks and the benefits of current and emerging weight-loss medications? Curr Diab Rep 9: 368-375.

25. Robert S, Chary SN, Drakakai G, Li S, Yang Z, et al. (2008) Endosidin1 defines a compartment involved in endocytosis of the brassinosteroid receptor BRI1 and the auxin transporters PIN2 and AUX1. Proc Natl Acad Sci U S A 105: 8464-8469.

26. Mauro M, Taylor V, Wharton S, Sharma AM (2008) Barriers to obesity treatment. Eur J Intern Med 19: 173-180. 